\title{
Feeling darkness: A visually induced somatosensory illusion
}

\author{
Uta Wolfe \\ Hobart and William Smith Colleges, Geneva, New York \\ AND \\ JaCob A. COMEe ANd BonNie S. SHERman \\ St. Olaf College, Northfield, Minnesota
}

\begin{abstract}
Although the five primary senses have traditionally been thought of as separate, examples of their interactions, as well as the neural substrate possibly underlying them, have been identified. Arm position sense, for example, depends on touch, proprioception, and spatial vision of the limb. It is, however, unknown whether position sense is also influenced by more fundamental, nonspatial visual information. Here, we report an illusion that demonstrates that the position sense of the eyelid partly depends on information regarding the relative illumination reported by the two eyes. When only one eye is dark-adapted and both eyes are exposed to a dim environment, the lid of the light-adapted eye feels closed or "droopy." The effect decreases when covering the eye by hand or a patch, thus introducing tactile information congruent with the interocular difference in vision. This reveals that the integration of vision with touch and proprioception is not restricted to higher-level spatial vision, but is instead a more fundamental aspect of sensory processing than has been previously shown.
\end{abstract}

Accurate knowledge of body position is crucial for motor control, because the central nervous system (CNS) needs to compare the actual to the intended position of the body for any goal-directed movement. This position sense derives from an interaction of spatial vision, touch, and proprioception (Botvinick \& Cohen, 1998; Farnè, Pavani, Meneghello, \& Làdavas, 2000; Graziano, 1999). Most of the studies on the relative roles of these modalities in position sense and on their neural representation have focused on the position sense of the arm or hand (e.g., Graziano \& Gross, 1994; Haggard, Newman, Blundell, \& Andrew, 2000). Although it has been demonstrated that the weighting of a given modality depends on its variability, such that crossmodal integration is driven by a statistical optimization function (Ernst \& Banks, 2002), most studies on position sense have reported that spatial vision of the limb generally predominates in the perception of the arm's position (Fink et al., 1999; Hay, Pick, \& Ikeda, 1965; Rock \& Victor, 1964). Thus, when vision and somatosensation give conflicting information, position sense is generally congruent with visual information and appears to ignore proprioceptive inputs. Goodwin, McCloskey, and Matthews (1972) have shown that vibrating the biceps tendon induces an illusion of position change of the forearm in blindfolded subjects, because somatosensory afferents are stimulated that normally convey a change in elbow flexion. However, the illusion disappears when subjects are allowed to see their arm, demonstrating that vision overrides proprioceptive signals.
Similarly, subjects seeing a rubber hand brushed - in synchrony with their own, hidden hand-report the illusion that tactile sensations derive from the rubber hand, that the fake hand feels as though it is their own, and that the perceived position of their own hand is distorted to match that of the seen rubber hand (Botvinick \& Cohen, 1998). Moreover, this effect is eliminated when vision indicates that the fake hand is in a posture not anatomically consistent with belonging to the subject's body (Pavani, Spence, \& Driver, 2000). Vision also appears to drive the kinesthetic and tactile sensations that are referred to as phantom limbs, and it possibly abolishes phantom limb pain by providing sensory feedback that might stop exaggerated central motor commands to the amputated limb (Ramachandran \& Rogers-Ramachandran, 1996).

Such cross-modal perceptual effects seem to arise from the correlation between visual and somatosensory inputs under normal conditions. The congruent visual and somatosensory sensations corresponding to a given arm position appear to be so firmly coupled that it is impossible to simultaneously experience visual sensations consistent with one arm posture and somatosensory sensations consistent with another. Instead, if the two sources of information are in conflict, the experience is driven by vision. The neural mechanism underlying this might involve the suppression of signals from muscle spindle afferents in cases in which visual and proprioceptive inputs are in conflict (Jones, Wessberg, \& Valbo, 2001).

U.Wolfe, uwolfe@hws.edu 
A connectionist neural model for these psychophysical cross-modality phenomena has been proposed (Botvinick $\&$ Cohen, 1998), and neural substrates possibly underlying them have been identified in the form of convergence of visual and somatosensory information onto multisensory cells in the superior colliculus (Meredith, 2002; Patton, Belkacem-Boussaid, \& Anastasio, 2002; Wallace \& Stein, 1997), but also in cortical areas such as premotor cortex, postcentral gyrus, parietal area $7 \mathrm{~b}$, and the ventral intraparietal area (Duhamel, Colby, \& Goldberg, 1998; Graziano, 1999; Graziano \& Gross, 1995; Iriki, Tanaka, \& Iwamura, 1996; Rizzolatti, Scandolara, Matelli, \& Gentilucci, 1981). These populations of cortical cells are thought to code for peripersonal space and to provide maps for the spatial control of movement (di Pellegrino, Làdavas, \& Farnè, 1997; Farnè et al., 2000; Graziano \& Gross, 1998). The consistent psychophysical observation that vision overrides proprioception when the two are discordant is to some extent reflected in the finding that the responsiveness of some visual-tactile cells to peripersonal visual stimuli decreases or vanishes when information on arm position is provided by proprioception alone (Graziano, 1999).

The preponderance of vision over somatosensation in the position sense is thus well documented in psychophysical observations and is partly mirrored in the neuronal response properties of multimodal cells. It has been suggested that vision's predominance is designed to compensate for the low spatial resolution of senses such as proprioception (Ernst \& Banks, 2002; Farnè et al., 2000), and thus far the studies showing this bias have concentrated on the role of spatial vision of the limb.

Here, we propose that for certain tasks, even nonspatial aspects of vision - such as information on the illumination level - interact with and dominate somatosensation in the derivation of position sense. Spatial vision of the eyelid is not available in the same form as is spatial vision of the limbs: For most natural eye positions, the border of the lid will fall either outside of the visual field or in the periphery, where spatial resolution is low. Moreover, even for eye positions in which the lid would be within foveal vision, spatial resolution at distances as close as the lid is restricted by the limits of accommodation (Koretz \& Handelman, 1988). Hence, spatial vision seems unlikely to be a reliable indicator of lid position. Similarly, although it is still being debated, there is evidence that the muscles moving the eye and lid lack classic mechanoreceptors and that sensory feedback on lid position through proprioception is limited (Fuchs, Becker, Ling, Langer, \& Kaneko, 1992; Lukas, Aigner, Blumer, Heinzl, \& Mayr, 1994; Matsuo, 2002; Porter, Burns, \& May, 1989; Ruskell, 1999; Trigo, Gruart, \& Delgado-Garcia, 1999). It therefore appears plausible that, rather than spatial vision or proprioception, information on illumination level, especially on the relative illumination level of the two eyes, might be used as an indicator of lid position. As is the case for arm position, there are constraints on the possible combinations of visual and somatosensory information regarding relative lid position under normal circumstances. Visual information indicating that one eye experiences less illumination than the other is normally accompanied by somatosensory information signaling occlusion of the "dim" eye, either by the lid or by an object that would give rise to tactile inputs. This regular intermodal association of sensory experiences might have induced a solid coupling of somatosensory and visual sensations similar to the one observed for arm position. Disrupting this correlation should then be expected to elicit perceptual illusions that reconcile the two senses at the cost of distorting one of them. If nonspatial vision is predominant in the position sense of the lid, the effect should be a distortion of somatosensation.

We tested this possibility in the series of experiments described below. By asymmetrically dark-adapting the eyes, we disrupted the normal association between somatosensation and visual information on the eyes' relative illuminations. We report that this dissociation elicits a distortion in lid position sense. This illusion is reduced in the presence of stimulation that satisfies the constraints on the possible combinations of visual, proprioceptive, and tactile information. Our results thus reveal an interaction of nonspatial vision with both proprioception and touch in the position sense of the eyelid. These findings indicate that cross-modal integration is not restricted to spatial vision but is a more fundamental feature of perceptual processing than has been previously shown.

\section{EXPERIMENT 1}

The first experiment examined whether disrupting the normal association between vision and somatosensation with respect to eyelid position would lead to perceptual distortions, and whether these distortions were driven by vision.

By dark-adapting only one eye and exposing both eyes to a dim environment, we induced a conflict between vision and somatosensation: Although vision signaled unequal illumination of the two eyes, somatosensation did not indicate an occlusion of either eye by the lid or a tactile stimulus. A strong coupling of normal visual and somatosensory sensations related to lid position should be expected to induce a perceptual distortion in one of the senses. If vision predominates in the interaction, the effect should be on somatosensation. We show that our method indeed induces a proprioceptive illusion that is driven by the asymmetry in lighting conditions reported by the two eyes.

\section{Method}

Subjects. Fourteen naive observers ( 6 male, 8 female) were recruited from the college's introductory psychology subject pool. All of them had normal vision or vision corrected to normal with contact lenses.

Procedure. All of the experiments took place in a windowless room $(4 \times 4 \times 2.5 \mathrm{~m})$. By regulating two sets of fluorescent lights, we varied light conditions in the room between four levels for the different stages of the experiment: Level 1, $70 \mathrm{fc}$; Level 2, $0.05 \mathrm{fc}$; Level 3, $0.005 \mathrm{fc}$, Level 4, $0.001 \mathrm{fc}$ (measured at the subject's eye level with a photometer, Tektronix J16, Illuminance Probe J6511).

Before the experiment, we showed the observers two nine-step visual analogue scales that they would use to indicate (1) the presence or absence of any perceptual effects (see scale shown in Figure 1), and (2) any change in the perceptual effects (see Figure 2). 


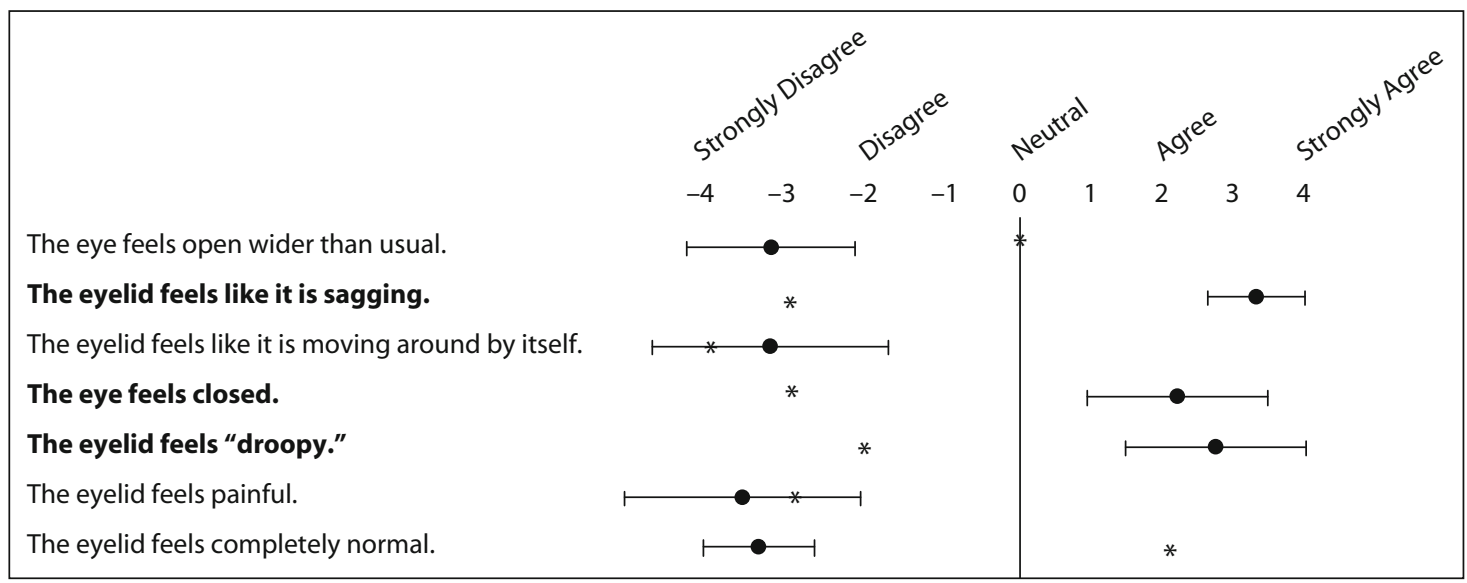

Figure 1. Average responses and standard deviations for the 13 subjects reporting the predicted perceptual effect. The responses by the outlier (the 1 subject not reporting the effect) are marked by an asterisk for each question. With or without the outlier, responses had a significant tendency to be positive for the bolded items and to be negative for the control items.

We induced asymmetric dark adaptation by first dark-adapting both eyes for $30 \mathrm{~min}$ (at Level 3) and then light-adapting one eye for $1 \mathrm{~min}$ (Level 1) while the subject closed the other eye (left, $n=7$; right, $n=7$ ) and covered it with a hand. After $1 \mathrm{~min}$, we darkened the room to Level 3 again and the subject uncovered and reopened the eye. ${ }^{1}$

An experimenter recounted a list of statements regarding the sensation in the eyelid of the light-adapted (LA) eye and asked the subjects to numerically rate their level of (dis)agreement with each statement. The statements included those describing the predicted phenomena ("The eyelid feels closed/droopy/like it is sagging") as well as several control statements ("The eyelid feels painful/completely normal/like it is moving around by itself"). To ensure that the perceptual phenomenon was restricted to the LA eye, we also asked the subjects whether they experienced any of the above sensations in the darkadapted (DA) eye. All of the subjects reported that the DA eyelid felt completely normal. As the subjects sat in the darkened room, the experimenter used infrared goggles to examine the lids of the first 4 subjects reporting the sensation of sagging in the lid, to ensure that the sensation was indeed illusory. It was thus confirmed that both eyes were open to the same degree even while the subject indicated a distinct feeling of sagging in the LA eye's lid. ${ }^{2}$

The subjects who reported the predicted phenomena participated in the second part of the experiment, in which illuminance was either increased or decreased to test whether this would prompt a change in the illusion. We hypothesized that both conditions would decrease the effect since both reduce the asymmetry between the eyes: Lowering illuminance would reduce the visual input from the DA eye (such that both eyes report "darkness"), whereas increasing illuminance would increase the input from the LA eye (such that both eyes can discern some visual stimuli).

Illuminance was reduced to Level 4 twice for $10 \mathrm{sec}$. Seven of the subjects also completed a second condition, in which illuminance was increased to Level 2 twice for $10 \mathrm{sec}$. In both conditions, the subjects were asked to use the second visual analogue scale to rate any change they experienced in the sensation during the periods when illumination was changed.

For each item, we calculated the confidence interval (CI) for the mean of the subjects' numerical responses. We thus tested whether the subjects' responses to an item had a significant tendency to be positive or negative by determining whether the CI around the sample mean was restricted to the positive or the negative range (and thus did not include zero).

\section{Results and Discussion}

All but 1 of the 14 observers reported the sensation of the eyelid of the LA eye as "sagging," "closed," or "droopy" (see Figure 1 for averages and standard deviations). The three statements for which our hypothesis predicted positive ratings are shown in bold. Even with the outlier included in the analysis, the responses to these three statements had a statistically significant tendency to be positive, whereas responses to the control items had a tendency to be negative $(p<.0001$ for each of the seven

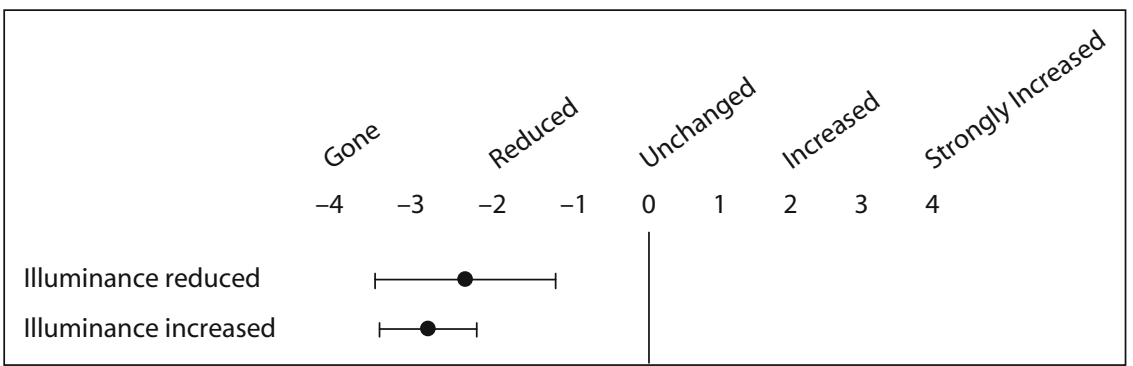

Figure 2. Average responses and standard deviations for all 13 subjects reporting the predicted perceptual effect. For both conditions, responses had a significant tendency to be negative, indicating the predicted decrease in illusion as the asymmetry in visual input from the eyes decreased. 
items; i.e., the $99.999 \%$ CI was contained in the positive range for the three test items, and in the negative range for the control items).

The second part of the experiment was designed to rule out the possibility that the unusual sensation was an artifact induced by the experimental method, which required the subjects to cover one eye for a brief period. If the sensation is indeed due to the asymmetry in lighting conditions reported by the eyes - and not to any asymmetry in tactile input required by the experimental method - then the sensation should decrease when the illumination is increased or decreased, because this would reduce the asymmetry in illumination reported by the eyes. As shown in Figure 2, the subjects tended to indicate a reduction or disappearance of the sensation when illumination was increased or decreased such that their numerical responses tended to be negative $(p<.0001)$.

These results thus confirm that the position sense of the eyelids is strongly influenced by the relative light intensity reported by the two eyes: When the eyes report unequal levels of illumination, the eyelids are perceived to be in unequal positions, so that the eye reporting less illumination is perceived to be (partly) closed. This finding is congruent with research on the position sense of the arm, which has been demonstrated to depend strongly on spatial vision of the limb, even at the cost of ignoring and distorting proprioception (Haggard et al., 2000). In our experiment, the influence of spatial vision was ruled out (because both eyelids were confirmed to be equally open), thus suggesting that even nonspatial vision interacts with and dominates somatosensation.

We hypothesized that the perceptual effect observed here belongs to the category of illusions that involves an obligatory coupling between specific, congruent inputs from vision, touch, and proprioception as well as an intersensory bias toward vision. Under normal circumstances, the most likely cause of unequal illumination of the two eyes would be closure or covering of one eye, which would give rise to congruent proprioceptive or tactile information from the occluded eye. Inducing the visual system to report asymmetric illumination of the eyes in the absence of congruent tactile or proprioceptive inputs, as our experimental task did, would disrupt this normal correlation between the senses and would - through visual dominance - induce the proprioceptive illusion we reported. This would predict that the illusion of the eye as closed should only persist in the absence of other somatosensory input that could explain the asymmetric visual information reported by the two eyes, and that would thus abolish the incongruity causing the illusion. This prediction would also be consistent with the commonplace observation that the illusion is not experienced when asymmetric vision is caused by simply wearing an eye patch or by covering the eye with a hand. We tested this model in Experiment 2.

\section{EXPERIMENT 2}

The illusion found in Experiment 1 appears to reconcile the senses of vision and somatosensation, because a closed lid is congruent with unequal visual input to the two eyes. It follows that the illusion should not arise if extraneous inputs were available that by themselves could explain the visual asymmetry. We tested this by first inducing the illusion and then asking subjects to close one eye actively or to cover it with a patch or by hand. We predicted that thus introducing tactile, proprioceptive, and/or motor corollary information congruent with asymmetric vision would decrease the incongruency between vision and somatosensation and would therefore lead to a reduction in the illusion.

\section{Method}

Subjects. The 13 observers who showed the predicted effect in Experiment 1 participated in Experiment 2. These subjects had not been debriefed following Experiment 1 and were thus naive to the purpose of the experiment.

Procedure. The observers were shown the nine-step visual analogue scale shown in Figures 2 and 3 on which they would indicate any change in sensation. Induction of the illusion occurred as described above. The subjects were then asked to close or cover (by hand or by patch) one eye at a time, as outlined in the six bolded conditions in Figure 3. All of these experimental conditions were predicted to decrease or abolish the effect. As control conditions hypothesized to have no effect on the sensation, we also asked subjects to (1) blink their eyes, (2) cover and then uncover their eyes, and (3) roll their eyes (see the nonbolded conditions in Figure 3).

For each condition, the subjects were asked to numerically rate any change in the illusion. For the condition in which subjects closed the LA eye actively, they were asked to indicate whether the unusual sensation of the eye as sagging or droopy disappeared, and not whether or not the eye felt closed.

\section{Results and Discussion}

As predicted, the six experimental conditions tended to reduce or eliminate the illusory effect, whereas the three control conditions did not. As seen in Figure 3, responses to the bolded items tended to be negative $(p<.0001)$, whereas those to the control items were not significantly different from zero $(p>.1)$.

The decrease in effect in Conditions 1, 3, and 5 (LA eye covered by $\mathrm{patch} / \mathrm{hand}$, closed actively) illustrates that introducing proprioceptive and/or motor corollary inputs congruent with the asymmetry in visual input reduces or eliminates the illusion. Visual asymmetry persists in these conditions: Because with or without the covering/ closing, the LA eye reports darker conditions than the DA eye, the reduction of the effect therefore appears to be due to the introduction of somatosensory and motor inputs alone.

In Conditions 2, 4, and 6 (DA eye covered or closed actively), the decrease in effect comes about both as visual information changes (the DA eye now reports conditions at least as dark as the LA eye), and as somatosensory and/ or motor inputs are congruent with an asymmetry in vision in which the DA eye reports less light than the LA eye. The visual information (DA eye as dark as or darker than LA eye) is thus congruent with the somatosensory information (DA eye covered or closed), and the illusion is reduced. As explained below, we investigate in a short final experiment whether this congruency between 


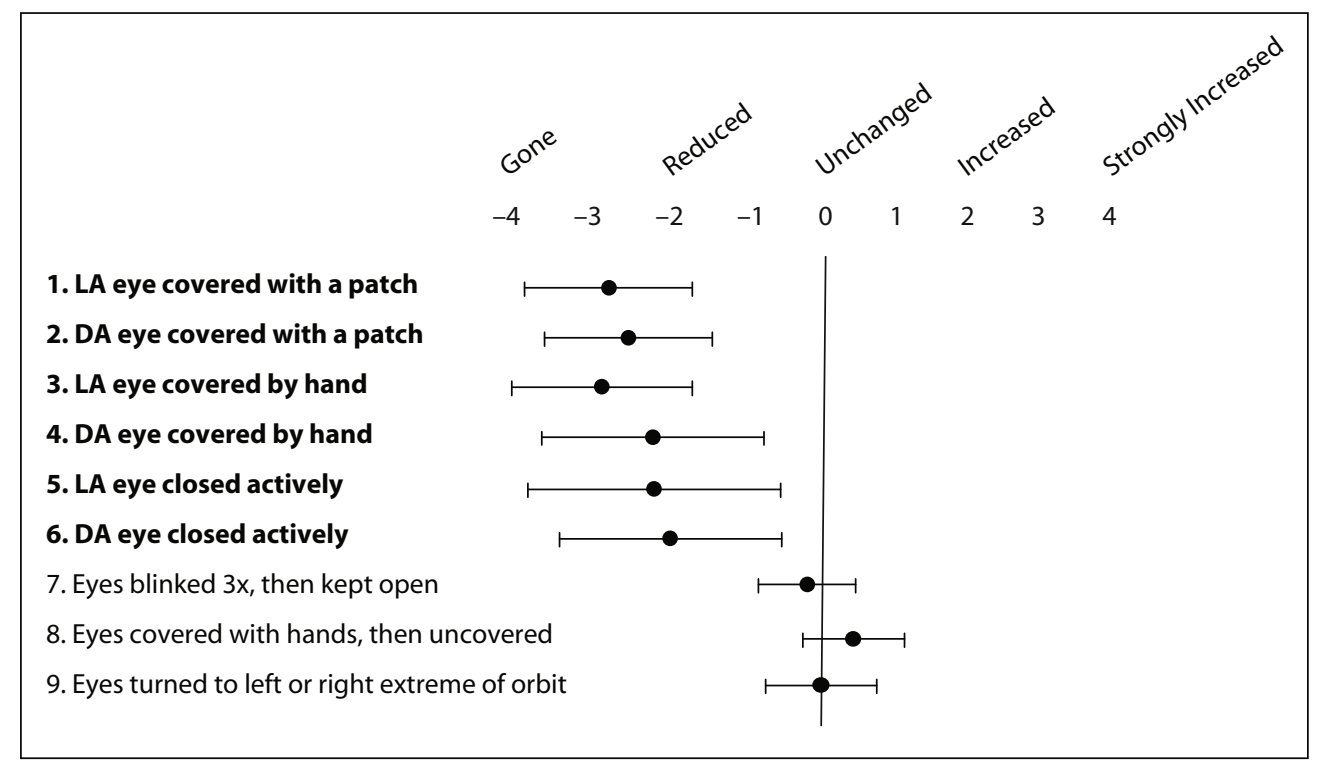

Figure 3. Average responses and standard deviations for all 13 subjects. Conditions shown in bold were predicted to lead to a decrease in illusion. As predicted, responses to the bolded items had a significant tendency to be negative, whereas responses to the control items were not significantly different from zero.

somatosensory input and the direction of visual asymmetry is necessary for the reduction of the illusion.

The results of Experiment 2 further support our theory that the illusion arises from a visually biased mechanism limiting the possible combinations of visual and somatosensory sensations, based on the normal correlation between these senses. The effect can thus be reduced by introducing tactile, proprioceptive, and/or motor corollary information that is consistent with the asymmetry in vision. We have thus demonstrated that visual information on the eyes' relative illumination is integrated and strongly coupled with both proprioception and touch in the subjective experience of eyelid position.

Our short final experiment addresses two questions arising from Experiment 2:

1. If vision is coupled with both touch and proprioception, why would the illusion arising from asymmetric vision be constrained to a proprioceptive effect? We propose that although the principal effect is the proprioceptive sensation of the eyelid as sagging or closed, more subtle tactile illusions might accompany the proprioceptive effects that we explicitly asked about in Experiment 1 . The different strengths of these effects might be a reflection of the difference in the coupling strengths between the pairs of modalities (vision/touch vs. vision/proprioception) that in turn arises from a difference in the frequency with which combinations of cross-modal inputs are experienced. In Experiment 3, we explore the possibility that part of the illusory effect might be on the modality of touch.

2. In Experiment 2, as indicated above, all of the conditions resulting in a reduction in illusion included somatosensory information that was consistent with the direction of visual asymmetry induced in the condition. Thus, the eye that was covered was also the eye reporting darker conditions. A model postulating that the illusion arises from the constraint that only normally occurring combinations of visual and somatosensory inputs can be experienced would predict that the effect can only be reduced when somatosensory input is congruent with the direction in asymmetry. However, it is also possible that somatosensory information signaling either eye's occlusion automatically terminates the comparison between the illumination of the left and right eyes, thus, in effect, telling the CNS to ignore any asymmetry in visual input, and thereby decreasing the illusion. We test this possibility in our final experiment.

\section{EXPERIMENT 3}

In order to test the hypothesis that at least part of the illusory effect is on the sense of touch, we asked naive subjects to give an open-ended description of their sensations involving the LA eye and lid, and we examined these reports for indications of haptic sensations.

To investigate whether, in order to decrease the illusion, somatosensation has to be consistent not only with the presence of visual asymmetry but also with its direction, we asked subjects to cover one of their eyes with a transparent disk, thus changing only the haptic, but not the visual, input to the eyes. When one is covering the LA eye, the haptic input is consistent with the direction of the visual asymmetry (i.e., the eye reporting darker conditions is covered). However, when one is covering the DA eye, haptic information is inconsistent with the direction of difference in visual inputs, because somatosensation signals occlusion of the eye reporting lighter conditions.

\section{Method}

Subjects. Seven naive subjects recruited from the college's introductory psychology subject pool participated in Experiment 3. 
Procedure. Before the experiment, the subjects were shown the scale in Figure 4 on which they would indicate any change in their sensation. We accomplished asymmetric dark adaptation as described above. The subject was asked to pay close attention to the lid of the LA eye and to give the experimenter an open-ended description of any sensations experienced. All 7 subjects indicated the predicted perceptual effect of the eyelid of the LA eye as "sagging," "closed," or "droopy" as part of their answer. They were then handed a 4-cm-diameter transparent plastic disk and asked to cover one eye at a time with the disk while leaving the eye open.

The subjects were not told that the disk was transparent (although they would presumably notice the transparency when covering the DA eye). They used the nine-step scale to rate the change in the sensation they were experiencing.

\section{Results and Discussion}

The subjects' open-ended descriptions of the sensations in the LA eye and lid revealed that they experienced tactile as well as proprioceptive sensations. Although all of the subjects indicated that the primary feeling was one of sagging in the lid, 6 of the 7 subjects included descriptions of the sensation as one of "pressure/touch" $(n=3)$, "tingling" $(n=1)$, "numbness" $(n=3)$, and/or "puffiness/ swelling" $(n=1)$ in the area surrounding the eye. This is consistent with our proposal that the origin of the illusion lies in the normal three-way interrelation among vision, proprioception, and touch. The finding that the tactile component of the illusion is notably weaker than the proprioceptive one might be due to a weaker coupling between vision and touch than between vision and proprioception in this context. It appears reasonable to assume that for most individuals, the frequency with which one eye is closed actively is higher than the frequency with which one eye is covered. Thus, asymmetric vision would be more strongly correlated with proprioception, indicating closure of the lid, than with touch, signaling occlusion of the eye.

The results for the second part of the experiment (shown in Figure 4) reveal that, whereas the illusion decreases when subjects cover the LA eye with the transparent disk, covering the DA eye has no effect on the illusion. The responses to covering the LA eye tended to be negative $(p<.0001)$. In contrast, responses to covering the DA eye were not significantly different from zero $(p>.1)$. This indicates that somatosensory inputs can only reduce the illusion if they are consistent with the direction of visual asymmetry so that they satisfy the usual correlation between the senses. The perceptual effect is decreased only when tactile inputs indicate that the eye reporting darker conditions is covered, but not when tactile information indicates occlusion of the eye reporting lighter conditions. This rules out the possibility of a mechanism by which somatosensory information indicating occlusion of either eye abolishes a comparison between the illumination levels of the eyes and thus reduces the illusion.

\section{GENERAL DISCUSSION}

We show a distortion in eyelid position sense that appears to originate from a coupling of nonspatial vision, proprioception, and touch. The parameters of this coupling seem to derive from the probability with which combinations of specific cross-modal sensations occur under normal circumstances. Consistent with the previously demonstrated predominance of spatial vision in the position sense of the arm, we report that the perceptual effect is driven by vision, so that asymmetric illuminance information from the eyes triggers illusory sensations of sagging in one lid (and to a lesser extent of tactile occlusion of one eye), thus satisfying the normal correlation between vision and somatosensation. The perceptual effect is reduced when extraneous tactile or motor corollary stimulation is introduced, which by itself can fulfill the constraints on the possible combinations of cross-modal sensations.

The cross-modal sensory impression therefore appears to be ruled by a Bayesian logic: A certain visual input will trigger the somatosensory sensation that in prior experience is most often coupled with the given visual information. Thus, asymmetric vision in normal experience is most probably caused by closure of one eye and rarely or never by a state of unequal sensitivity of the eyes. This explanation is similar to the one offered by Ramachandran and Hirstein (1998) for several unusual perceptual effects, such as their observation that a blindfolded subject will perceive a dislocation of his or her nose when identical sequences of touches are simultaneously given to the subject's nose by an experimenter and to another person's nose with the subject's own finger. A Bayesian model would predict that the sensory system would interpret the touch

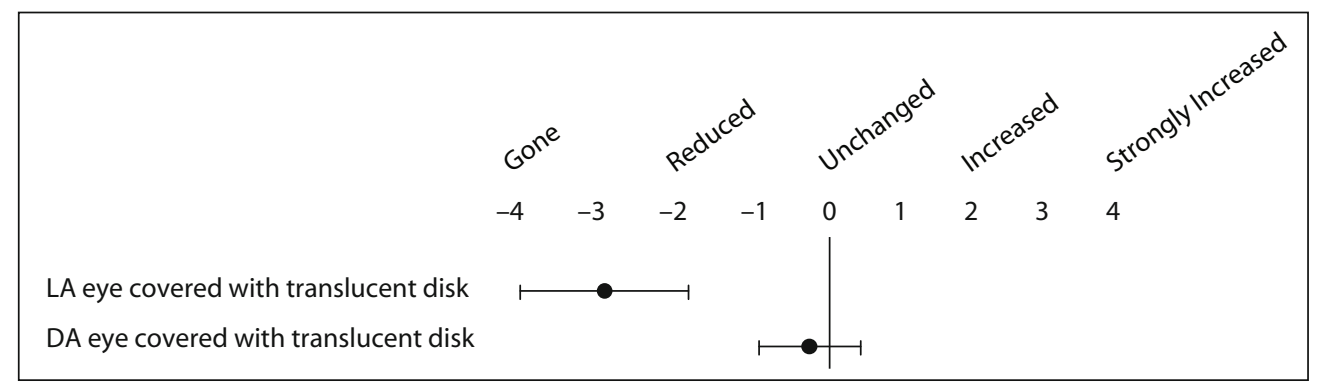

Figure 4. Average responses and standard deviations for all 7 subjects. Covering the LA eye with a transparent disk led to a decrease in illusion, whereas covering the DA eye did not significantly reduce the effect. 
to the nose and to the finger as originating from a unified event because of the statistical improbability of two perfectly identical tactile sequences arising from two separate events. This model would also apply to the findings by Botvinick and Cohen (1998) that we described earlier.

A neural model for such a Bayesian-driven interaction between different modalities might include inputs from different senses converging onto a single multisensory neuron that through Hebbian learning develops into a "labeled line" for a specific combination of cross-modal inputs. In our case, activity in such a neuron would signal a pattern of interocular illumination asymmetry, coupled with occlusion of one eye, thus giving rise to a specific percept, such as "right eye dark and closed." The neural substrate for this cross-modal perception might entail the superior colliculus, which receives both bilateral projections from "luminance detectors" in the olivary pretectal nucleus (involved in the pupillary light reflex; Klooster, Vrensen, Müller, \& van der Want, 1995) and from primary afferents of the eyelid (VanderWerf, Buisseret-Delmas, \& Buisseret, 2002). Activating this labeled line through vision alone would still give rise to the cross-modal percept and thus to the illusory somatosensation. A similar explanation involving the "switching" of labeled lines has been offered for the occurrence of phantom limb sensation (Ramachandran \& Hirstein, 1998). Assuming that such Bayesian-driven Hebbian learning underlies much of our sensory processes, the involvement of vision in eyelid position sense, rather than having an adaptive function, might be a side effect of a ubiquitous mechanism causing the coupling of cross-modal sensations that are highly correlated in normal experience.

The synaptic strength between the input neurons and the putative multisensory neuron - and thus, the coupling strength between the sensory modalities - would depend on the relative frequency with which specific cross-modal combinations of inputs occur. This might explain why the illusory effect described here was primarily proprioceptive rather than tactile, if we assume that visual asymmetry is most often caused by active lid closure rather than by tactile covering of the eye. It would also be consistent with Barlow's statistical inference model, by which neural responses reflect the probability of a given stimulus (Barlow, 1969,1972 ), or with the more recent findings (Anastasio, Patton, \& Belkacem-Boussaid, 2000; Patton et al., 2002) that response properties of multisensory neurons in the superior colliculus follow Bayes's rule.

One question arising from our findings is that of how stable the Bayesian-ruled coupling of sensations is, and how much experience with unusual combinations of sensory inputs would be necessary to change the coupling strengths. In our study, we found that the illusory sensation began to fade 5-8 min after its induction. Given our experimental method, this fading was more likely caused by the gradual dark adaptation of the LA eye (and thus by the reduction in visual asymmetry) and possibly by adaptation of the putative "labeled line" neurons, rather than by a shift in coupling strength. We are currently preparing a study in which subjects wear a dark contact lens that completely blocks light from entering one eye, so that the time course of decay in the illusion can be measured without the compounding factor of dark adaptation. This also allows us to investigate whether repeated or prolonged wearing of the lens reduces even subjects' initial experience of the illusion, as would be predicted by a reorganization of sensory coupling according to Bayes's rule. ${ }^{3}$

The finding that extraneous stimulation consistent with visual asymmetry reduces the illusory proprioceptive sensation seems to suggest an inhibitory influence of somatosensory inputs on the activity of multisensory neurons coding for the cross-modal percept. This might be related to the phenomenon of reweighting of different modalities' inputs, which has been demonstrated in the control of posture (Oie, Kiemel, \& Jeka, 2002), and for tasks involving visual-haptic judgments of extent (Ernst \& Banks, 2002). This work shows that changes in the amplitude (Oie et al.) or variability (Ernst \& Banks) of visual or tactile inputs lead to a reweighting of the modalities. Similarly, the tactile inputs in our study might reduce the illusion by a decrease in the weight given to the multisensory neurons' input, because the extraneous touch gives rise to a more reliable indicator of lid position. This would be consistent with the model by Oie and colleagues, in which multisensory fusion is a nonlinear process through which sensory inputs are dynamically reweighted, presumably according to their degree of reliability.

\section{AUTHOR NOTE}

We thank Jason Forte, Laurence Maloney, and Heather Munro for critical reading of the manuscript. Correspondence concerning this article should be addressed to U. Wolfe, Hobart and William Smith Colleges, Psychology Department, Geneva, NY 14456 (e-mail: uwolfe@hws.edu).

\section{REFERENCES}

Anastasio, T. J., Patton, P. E., \& Belkacem-Boussaid, K. (2000). Using Bayes' rule to model multisensory enhancement in the superior colliculus. Neural Computation, 12, 1165-1187.

BARLOW, H. B. (1969). Pattern recognition and the responses of sensory neurons. Annals of the New York Academy of Sciences, 156, 872-881.

BARLOW, H. B. (1972). Single units and sensation: A neuron doctrine for perceptual psychology? Perception, 1, 371-394.

Botvinick, M., \& CoHEN, J. (1998). Rubber hands "feel" touch that eyes see. Nature, 391, 756.

di Pellegrino, G., Làdavas, E., \& Farnè, A. (1997). Seeing where your hands are. Nature, 288, 730.

Duhamel, J. R., Colby, C. L., \& Goldberg, M. E. (1998). Ventral intraparietal area of the macaque monkey: Congruent visual and somatic response properties. Journal of Neurophysiology, 79, 126-136.

ERnst, M. O., \& BANKs, M. S. (2002). Humans integrate visual and haptic information in a statistically optimal fashion. Nature, 415, 429433.

Farnè, A., Pavani, F., Meneghello, F., \& LÀdavas, E. (2000). Left tactile extinction following visual stimulation of a rubber hand. Brain, 123, 2350-2360.

Fink, G. R., Marshall, J. C., Halligan, P. W., Frith, C. D., Driver, J., FraCKOWIAK, R. S. J., \& Dolan, R. J. (1999). The neural consequences of conflict between intention and the senses. Brain, 122, 497-512.

Fuchs, A. F., Becker, W., Ling, L., LANGer, T. P., \& Kaneko, C. R. (1992). Discharge patterns of levator palpebrae superioris motoneurons during vertical lid and eye movements in the monkey. Journal of Neurophysiology, 68, 233-243.

Goodwin, G. M., McCloskey, D. I., \& Matthews, P. B. (1972). The contribution of muscle afferents to kinaesthesia shown by vibration induced illusions of movement and by the effects of paralysing joint afferents. Brain, 95, 705-748.

GraZiano, M. (1999). Where is my arm? The relative role of vision and 
proprioception in the neuronal representation of limb position. Proceedings of the National Academy of Sciences, 96, 10418-10421.

Graziano, M., \& Gross, C. (1994). Coding of visual space by premotor neurons. Science, 266, 1054-1057.

GraZiano, M., \& Gross, C. (1995). The representation of extrapersonal space: A possible role for bimodal, visual, tactile neurons. In M. S. Gazzaniga (Ed.), The cognitive neurosciences (pp. 1021-1034). Cambridge, MA: MIT Press.

Graziano, M., \& Gross, C. (1998). Spatial maps for the control of movement. Current Opinion in Neurobiology, 8, 195-201.

Haggard, P., Newman, C., Blundell, J., \& Andrew, H. (2000). The perceived position of the hand in space. Perception \& Psychophysics, 62, 363-377.

HAY, J. C., PicK, H. L., \& Ikeda, K. (1965). Visual capture produced by prism spectacles. Psychonomic Science, 2, 215-216.

Iriki, A., TANaKa, M., \& Iwamura, Y. (1996). Coding of modified body schema during tool use by macaque postcentral neurones. NeuroReport, 7, 2325-2330.

Jones, K. E., Wessberg, J., \& Valbo, A. (2001). Proprioceptive feedback is reduced during adaptation to a visuomotor transformation: Preliminary findings. NeuroReport, 12, 4029-4033.

Klooster, J., Vrensen, G. F., Müller, L. J., \& Van der Want, J. J. (1995). Efferent projections of the olivary pretectal nucleus in the albino rat subserving the pupillary light reflex and related reflexes: A light microscopic tracing study. Brain Research, 688, 34-46.

Koretz, J. F., \& Handelman, G. H. (1988). How the human eye focuses. Scientific American, 259, 92-99.

lukas, J. R., Aigner, M., Blumer, R., Heinzl, H., \& Mayr, R. (1994). Number and distribution of neuromuscular spindles in human extraocular muscles. Investigative Ophthalmology \& Visual Science, 35, 4317-4327.

Matsuo, K. (2002). Stretching of the Mueller muscle results in involuntary contraction of the levator muscle. Ophthalmic Plastic \& Reconstructive Surgery, 18, 5-10.

Meredith, M. A. (2002). On the neuronal basis for multisensory convergence: A brief overview. Cognitive Brain Research, 14, 31-40.

Oie, K. S., Kiemel, T., \& JeKA, J. J. (2002). Multisensory fusion: Simultaneous re-weighting of vision and touch for the control of human posture. Cognitive Brain Research, 14, 164-176.

Patton, P., Belkacem-Boussaid, K., \& Anastasio, T. J. (2002). Multimodality in the superior colliculus: An information theoretic analysis. Cognitive Brain Research, 14, 10-19.

Pavani, F., Spence, C., \& Driver, J. (2000). Visual capture of touch: Out-of-the-body experiences with rubber gloves. Psychological Science, 11, 353-359.
Porter, J. D., Burns, L. A., \& MAY, P. J. (1989). Morphological substrate for eyelid movements: Innervation and structure of primate levator palpebrae superioris and orbicularis oculi muscles. Journal of Comparative Neurology, 287, 64-81.

RamachandRan, V. S., \& Hirstein, W. (1998). The perception of phantom limbs. Brain, 121, 1603-1630.

Ramachandran, V. S., \& Rogers-Ramachandran, D. (1996). Synaesthesia in phantom limbs induced with mirrors. Proceedings of the Royal Society of London: Series B, 263, 377-386.

Rizzolatti, G., Scandolara, C., Matelli, M., \& Gentilucci, M. (1981). Afferent properties of periarcuate neurons in macaque monkeys: II. Visual responses. Behavioural Brain Research, 2, 147-163.

RocK, I., \& VICTOR, J. (1964). Vision and touch: An experimentally created conflict between the two senses. Science, 143, 594-596.

Ruskell, G. L. (1999). Extraocular muscle proprioceptors and proprioception. Progress in Retinal \& Eye Research, 18, 269-291.

Trigo, J. A., Gruart, A., \& Delgado-Garcia, J. M. (1999). Role of proprioception in the control of lid position during reflex and conditioned blink responses in the alert behaving cat. Neuroscience, 90, 15151528.

VanderWerf, F., Buisseret-Delmas, C., \& Buisseret, P. (2002). The afferent innervation of eyelids and their connections to the superior colliculus. Movement Disorders, 17, S8-S11.

Wallace, M. T., \& Stein, B. E. (1997). Development of multisensory neurons and multisensory integration in cat superior colliculus. Journal of Neuroscience, 17, 2429-2444.

\section{NOTES}

1. We chose this procedure over dark-adapting one eye by covering it for an extended time in order to minimize any asymmetries in tactile inputs to the two eyes, which might induce somatosensory artifacts.

2 . The results of Experiment 2 further confirm the perception as illusory, because it disappears or is reduced when the eye is covered. It is unlikely that a real droop in the LA eye's lid would disappear when the eye still reports darkness but is covered.

3. Similarly, as pointed out by one anonymous reviewer, patients with ptosis might not experience the illusion the same way as do the observers in our experiment. The patients' usual experience is that of asymmetric illumination of the eyes. One might speculate that if the ptotic lid were suddenly returned to normal, a patient might experience the illusion of drooping in the healthy lid.

(Manuscript received December 26, 2003; revision accepted for publication December 15, 2006.) 